\title{
Review Article \\ Review of Research on Template Methods in Preparation of Nanomaterials
}

\author{
Yadian Xie, ${ }^{1,2}$ Duygu Kocaefe, ${ }^{1}$ Chunying Chen, ${ }^{2}$ and Yasar Kocaefe ${ }^{1}$ \\ ${ }^{1}$ University of Quebec at Chicoutimi (UQAC), Chicoutimi, QC, Canada G7H2B1 \\ ${ }^{2}$ CAS Key Laboratory for Biomedical Effects of Nanomaterials and Nanosafety, National Center for Nanoscience and Technology, \\ Beijing 100190, China
}

Correspondence should be addressed to Yasar Kocaefe; yasar_kocaefe@uqac.ca

Received 26 January 2016; Revised 11 June 2016; Accepted 28 June 2016

Academic Editor: Shu Seki

Copyright (C) 2016 Yadian Xie et al. This is an open access article distributed under the Creative Commons Attribution License, which permits unrestricted use, distribution, and reproduction in any medium, provided the original work is properly cited.

\begin{abstract}
The nanomaterials have been widely used in various fields, such as photonics, catalysis, and adsorption, because of their unique physical and chemical properties. Therefore, their production methods are of utmost importance. Compared with traditional synthetic methods, the template method can effectively control the morphology, particle size, and structure during the preparation of nanomaterials, which is an effective method for their synthesis. The key for the template method is to choose different templates, which are divided into hard template and soft template according to their different structures. In this paper, the effects of different types of templates on the morphology of nanomaterials during their preparation are investigated from two aspects: hard template and soft template, combined with the mechanism of action.
\end{abstract}

\section{Introduction}

Material is a necessity for human life and production and a driving force that promotes the development of modern civilization. With the development of science and technology, the requirements for the material quality are increasingly demanding. Nanomaterials have a small size, between 1 and $100 \mathrm{~nm}$, and possess unique optical, electrical, magnetic, thermal, catalytic, and mechanical properties, which have been widely used in photonics, spectroscopy, industrial catalysis, biology, and other fields [1].

Nanomaterials have distinguished surface effect, volume effect, quantum size effect, macroscopic quantum tunneling effect, and dielectric confinement effect, which have attracted the attention of researchers. The research on preparation of nanomaterials has become a current hotspot. The common methods for preparing nanomaterials consist of physical and chemical methods [2-4]. Physical methods include physical pulverization method, physical coacervation method, and spray method. Chemical methods include chemical vapor deposition method, chemical precipitation, sol-gel method, hydrothermal method, and template method.
Template synthesis of nanomaterials has been a cuttingedge technology developed since the 1990s. It is also a very effective nanomaterials synthesis method that has been widely used in recent years. Early in 1999, South Korean scientists Kim et al. took MCM-48 mesoporous silica as template and synthesized three-dimensional cubic mesoporous carbon CMK-1 [5]. In the same year, Japanese scientists Moriguchi et al. used cationic surfactant $\mathrm{CTAB}$ as template and synthesized disordered carbon materials by using phenolic resin as carbon source [6]. Template method is not sensitive regarding the preparation conditions, easy to operate and implement, which controls the structure, morphology, and particle size of nanomaterials through the template material (template). Template method is often divided into hard and soft template methods. Dong et al. [7] from Fudan University adopted hard template method. Through using mesoporous carbon pellets as a template, they have synthesized a variety of nonsilicon-based mesoporous materials such as solid or hollow aluminum oxide, titanium oxide, and aluminum phosphate microspheres. Firouzi et al. [8,9] from the University of California used soft template method to explore a variety of different types of inorganic and organic combinations 
and proposed the general synthetic route to mesoporous materials.

Morphology is an important parameter for characterization of material properties, especially in the mesoporous material. Morphology, together with particle size, surface area, and pore structure, determines the property of mesoporous materials and, consequently, pinpoints their application $[10,11]$. Among this, mesoporous thin films, as filmlike material, have an unparalleled advantage in adsorption and separation compared to the other nanomaterials with different shapes [12,13].

The template method changes the morphology of products, mainly, by controlling the crystal nucleation and growth during nanomaterial preparation. Route of nanomaterial synthesis by using the template method is generally divided into three steps: First, the template is prepared. Second, some common synthetic approach such as hydrothermal method, precipitation, and sol-gel method is used to synthesize the target production under the function of template. Finally, the third step is the template removal. The choice of template for nanomaterial preparation is critical. Templates usually can be grouped in two major categories: natural substances (nanomineral, biological molecules, cells and tissues, etc.) and synthetic materials (surface active agents, porous materials and nanoparticles, etc.). In addition, the templates are generally divided into two groups (hard template and soft template) based on the difference in the template structure $[14,15]$.

Template removal is the last step in the synthesis of nanomaterials. The appropriate method of removal has to be chosen such that the physical and chemical properties of the product should not be affected. Common removal methods include physical and chemical methods, such as dissolution, sintering, and etching. In this paper, the control of morphology is illustrated while preparing nanomaterials by using different hard and soft templates combined with the mechanism.

\section{Hard Template}

Hard template is a rigid material, whose stable structure directly determines the size and morphology of sample particle. A wide choice of hard templates is available, such as polymer microspheres, porous membrane, plastic foam, ion exchange resin, carbon fiber, and porous anodic aluminum oxide (AAO) $[16,17]$. Because of their special structures and effect on particle size restriction, they play an important role in many fields.

2.1. Porous Anodic Aluminum Oxide (AAO). Porous anodic alumina has high pore density. The pores are evenly distributed and very thin. The pore size is adjustable in range of $50-200 \mathrm{~nm}$ and the pore density is between $1 \times 10^{9}$ and $1 \times 10^{12} \mathrm{~cm}^{-2}[18,19]$. It is widely used in the preparation of nanofunctional devices. For example, it is used in the deposition of metal, semiconductor, conductive polymer, and other functional materials and has been successfully applied

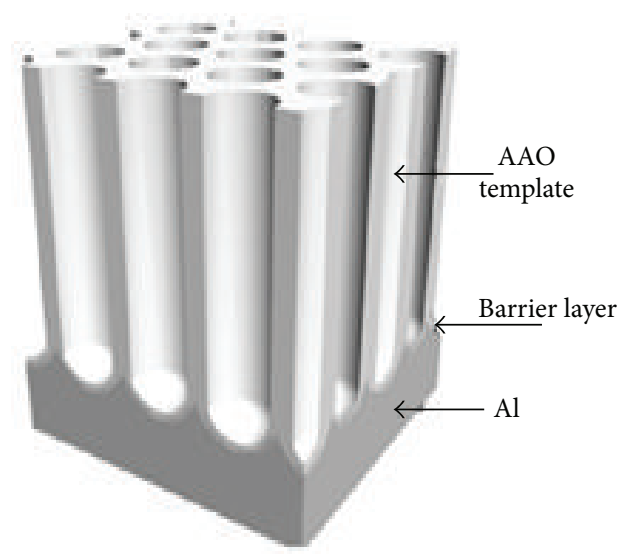

Figure 1: Structure diagram of AAO [24].

in fields of magnetic recording materials, optoelectronic components, electronics, and so forth [20-28].

2.1.1. Preparation of Porous Anodic Aluminum Oxide (AAO). The preparation process of AAO has not been unified. Usually the high purity aluminum piece is placed into an acidic solution, and AAO is obtained by electrolysis by the anodic oxidation. By changing anode voltage and the composition and concentration of electrolyte, the size and distribution of the pore are controlled. The structure diagram of AAO is shown in Figure 1. It can be seen from this figure that there is dense alumina called the barrier layer between the nonoxidized aluminum matrix composite and the porous layer. Since the barrier layer hinders the contact between the electrolyte solution and the electrode, this layer needs to be removed during the preparation of the sample by electrodeposition. The aluminum matrix composite also has to be removed in order to obtain the double pass AAO template.

\subsubsection{Application of Porous Anodic Aluminum Oxide (AAO).} Porous anodic alumina is widely used in the preparation of nanomaterials because of the adjustability and quantum level of pore size, such as in the preparation of onedimensional nanomaterials (nanowires, nanotubes), zerodimensional materials (nanodots), and mesoporous films. The method of preparing nanomaterials by using porous anodic aluminum oxide as template is divided into two groups: one, electrochemical deposition; two, deposition without electrolysis. The precursor is deposited in the inner hole of the AAO template, and it controls the structure of the nanomaterial by guiding the spatial restriction of the cylindrical bore arranged in matrix. The morphology of the particle we obtain is similar to that of the AAO template. Sun et al. [29] used AAO as the template and the electrochemical deposition method to prepare the $\mathrm{ZnS}$ nanowires with a diameter of $30 \mathrm{~nm}$, as shown in Figure 2(a). Lu et al. [30] synthesized rodlike mesoporous silica by using polypropylene glycol and P123 as surfactant and silica as precursor in the AAO template, which is shown in Figure 2(b). Forrer et al. [31] prepared gold nanowire arrays by using AAO template 


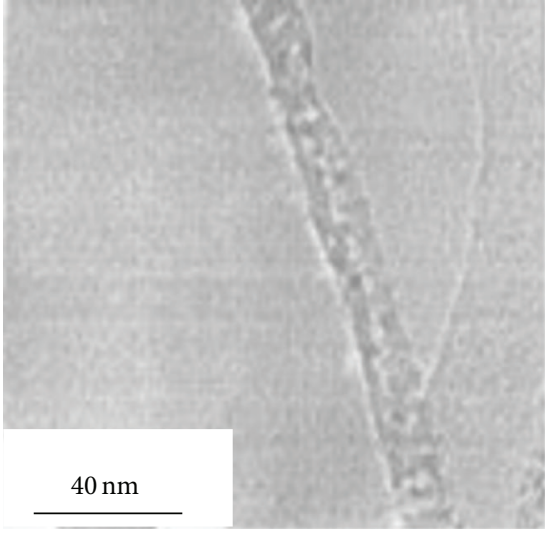

(a)

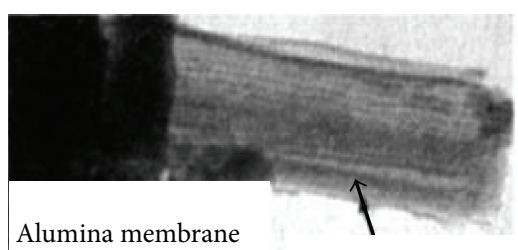

SBA-15 nanorods

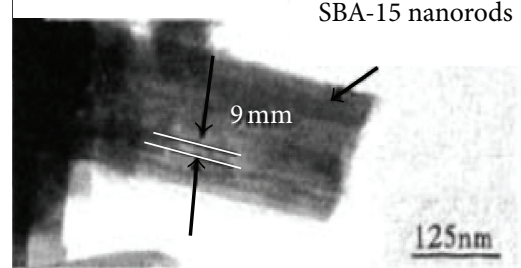

(b)

FIGURE 2: TEM image of products fabricated by AAO template. (a) ZnS nanowires. (b) Rodlike silica [25, 26].

technique. The synthetic process schematically demonstrates how the AAO template produces gold nanowires which is given in Figure 3. Figure 3(a) shows an AAO template with subsequent pores and Figure 3(b) shows gold nanoparticles were deposited in the pores by applying the AAO template potential in a gold bath. Figure 3(c) shows the alumina and the aluminum base metal were removed in a $\mathrm{NaOH}$ solution to expose a free standing array of nanowires.

2.2. Mesoporous Carbon. Mesoporous carbon is an important member of mesoporous materials. Its pore distribution is uniform and the pore diameter is generally between $2 \mathrm{~nm}$ and $50 \mathrm{~nm}$. It has regular pore structure and high specific surface area. It is also thermally and chemically stable. Mesoporous carbon materials are widely used in many fields such as hydrogen storage, adsorption, catalysis, batteries, and capacitors [32-38].

2.2.1. Synthetic Methods of Mesoporous Carbon. Synthetic methods of mesoporous carbon can be divided into catalytic activation, organic sol-gel method and template method, and so forth. Catalytic activation is used to synthesize mesoporous carbon through the catalytic effect of metals and their compounds act on carbon gasification [39, 40]. The activation reaction mainly occurs around the metal particles, which can inhibit the formation of micro pores and increase the mesopores [41]. Organic sol-gel method was proposed by Pekala and Schaefer [42]. This method uses the gel formed by the polymerization of precursor as carbon source, and the mesoporous carbon is obtained by high-temperature carbonization. Zhang et al. [43, 44] mixed melamine and formaldehyde with $\mathrm{NaCO}_{3}$ as catalyst and added mixed solution of phenol and formaldehyde and finally phenolic resin to get organic moisture gel. Finally, mesoporous carbon is obtained by carbonization. The structure of mesoporous carbon is shown in Figure 4 [45].

2.2.2. Application of Mesoporous Carbon. As the pore diameter of mesoporous carbon is even and the structure is

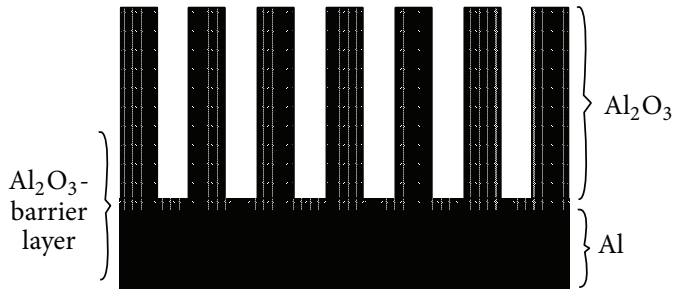

(a)

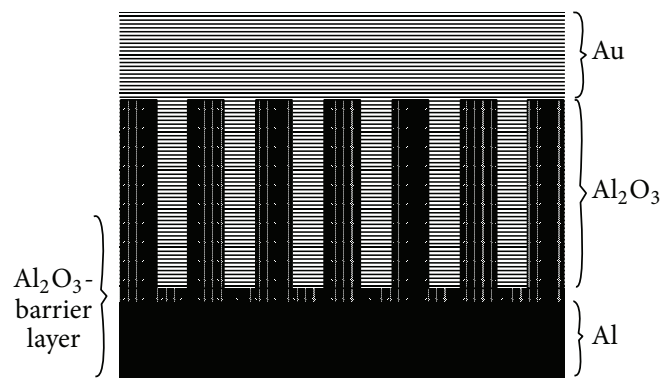

(b)

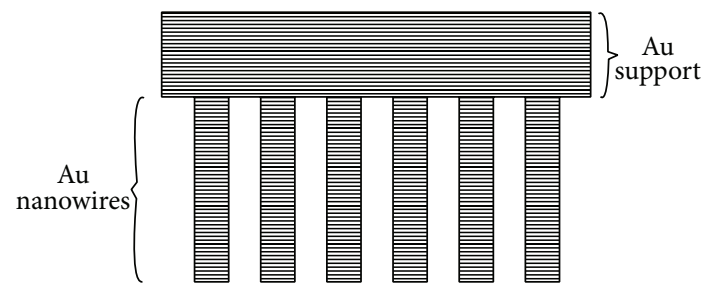

(c)

FIGURE 3: Synthesis of gold nanowires using porous aluminum oxide membrane template [27].

ordered, it is often used as template to synthesize ordered mesoporous materials such as nanozeolite molecular sieve and mesoporous metal oxides. In the synthesis of mesoporous materials, the mesoporous carbon pores are copied to a new mesoporous material by means of the nanoreplication technology, so the morphology and structure of 

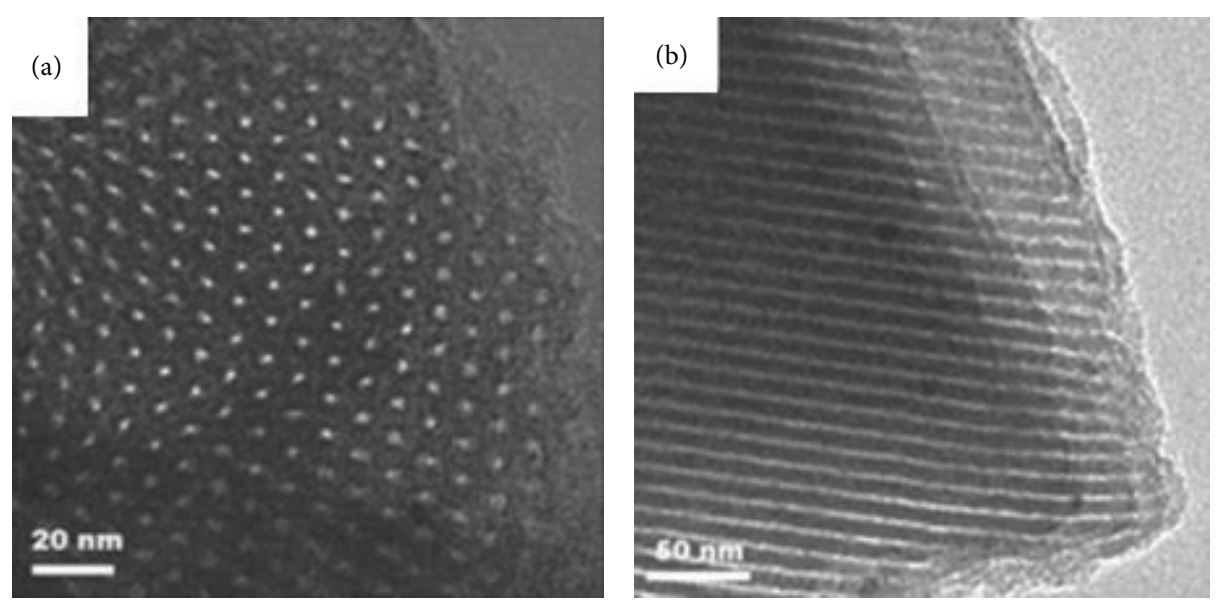

FIGURE 4: TEM image of mesoporous carbon structure [35].

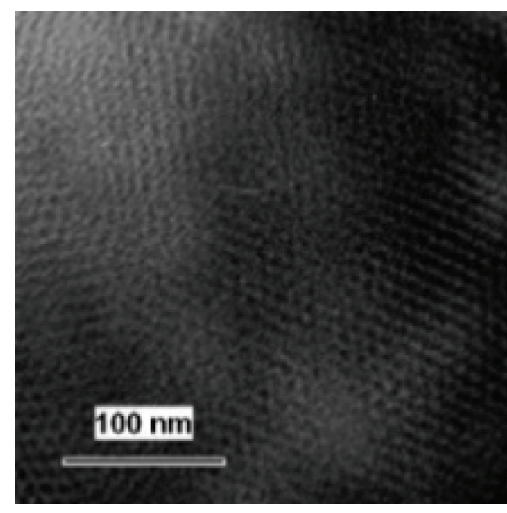

(a)

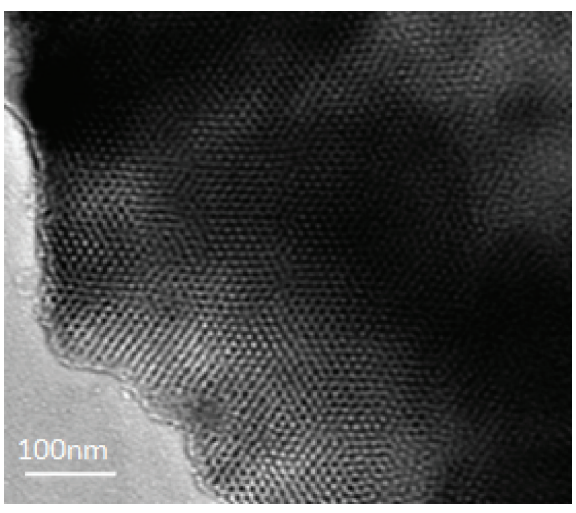

(b)

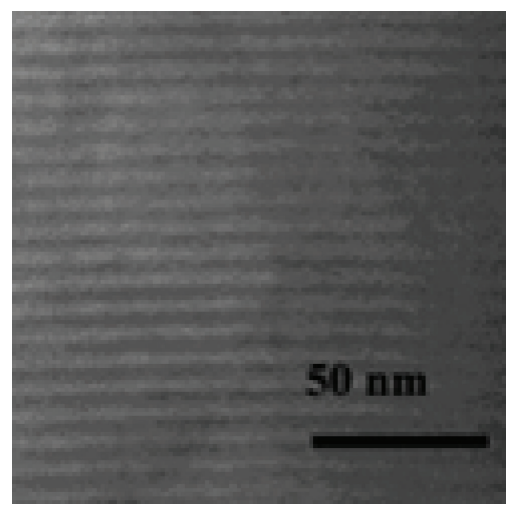

(c)

FIGURE 5: TEM image of particles prepared by CMKs-template. (a) Hexagonal mesoporous MgO. (b) RMM-1 with cubic crystal system. (c) RMM-3 with the hexagonal crystal system [37-39].

the particles obtained by this method are similar to those of the mesoporous carbon. Roggenbuck and Tiemann [46] and Roggenbuck et al. [47] synthesized successfully the hexagonal ordered mesoporous $\mathrm{MgO}$ by using mesoporous carbon CMK-3 as the template, as shown in Figure 5(a). Sakthivel et al. [48] used carbon mesoporous molecular sieve (CMKs) as the template to synthesize mesoporous zeolite molecular sieve. Yang et al. [49] synthesized the aluminosilicate molecular sieve RMM-1 with the cubic crystal system by CMK-1, which is shown in Figure 5(b), and aluminosilicate molecular sieve RMM-3 with the hexagonal crystal system by CMK-3, as shown in Figure 5(c).

In the preparation of nanomaterials by using mesoporous carbon or other porous materials as the template, the precursor tends to crystallize inside the pores or deposit outside the pores. When the diameter of the pore is larger than that of the primary unit cell of the material, the space of the pore will accommodate the unit cell, forming a crystal material which has the similar structure to the pore. On the contrary, when the diameter of the pore is smaller than the primary unit cell unit, the crystal tends to form core and grow between the pores and finally become irregular crystalline material.
2.3. Polymer Microspheres. The methods of synthesizing polymer microspheres are methods such as emulsion polymerization, microemulsion polymerization, soap-free emulsion polymerization, suspension polymerization, and dispersion polymerization. By controlling the rate of polymerization, the particle size can be controlled. Zhang et al. [50] have studied the effects of various factors (such as dispersion stabilizer, monomer, initiator, reaction medium polarity, reaction temperature, and agitation speed) on the particles, successfully synthesizing the polystyrene microspheres whose particle size is in the range of $1 \mu \mathrm{m}$ to $10 \mu \mathrm{m}$.

The polymer microsphere has a good dispersivity and an easily adjustable particle size. It is usually used to synthesize the spherical-shaped particles or near-spherical core-shell and the hollow structure after modifying the surface of the particles. Lin [51] synthesized the compound of PSA/ZnS/CdS core-shell structure by using homemade polystyrene-acrylic microspheres (PSA) as a template using the dip method. Then, the template PSA was removed with toluene solution, finally obtaining $\mathrm{ZnS} / \mathrm{CdS}$ composite hollow microspheres, whose microsphere size can be regulated by the amount of emulsifier. Figure 6 


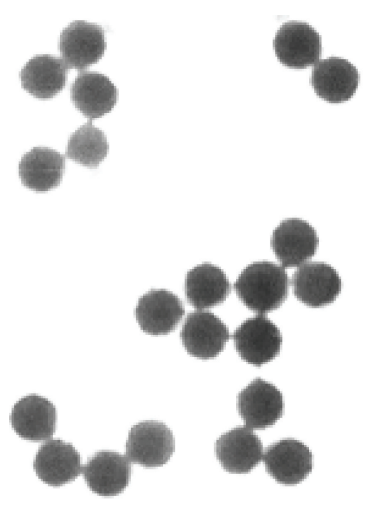

(a)

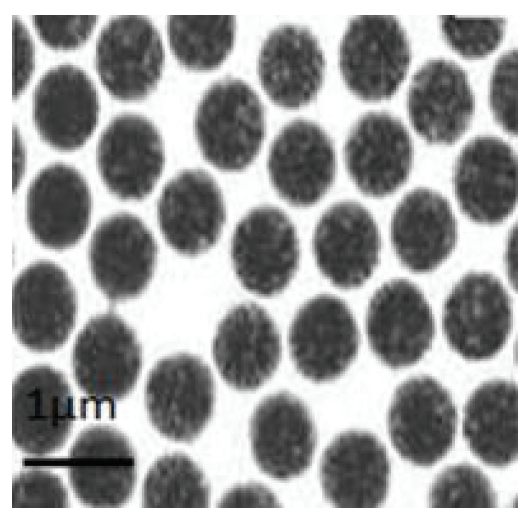

(b)

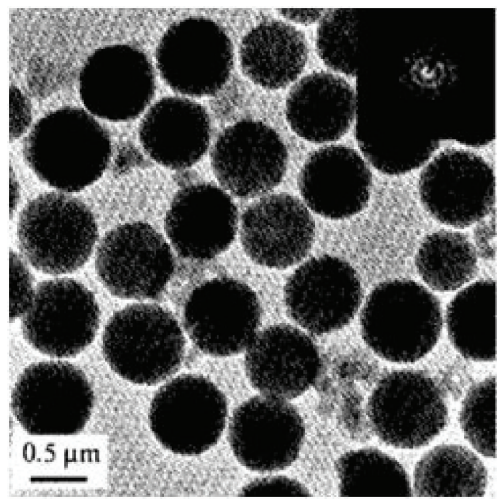

(c)

FIgURE 6: TEM image of ZnS/CdS composite hollow microspheres prepared by PSA. (a) PSA. (b) PSA/ZnS/CdS. (c) ZnS/CdS [41].

shows the particle morphology during various preparation stages.

2.4. Summary. Hard template is a commonly used method for the synthesis of nanostructured materials such as nanoparticles, nanorods, nanowire, nanotubes, and nanobelts in recent years. Because the method is capable of preparing templates with different nanohole size and structure under various requirements, using physical or chemical growth or deposition of nanomaterials in nanopores for nanomaterials fabrication, the dimensions and specifications of target production can be precisely controlled.

Because of the stability of hard template structure, it is often used as the "micro reactor" in synthesis, during which a precursor is filled into the pores of the hard template or absorbed at its surface by impregnation or chemical vapor deposition method. The special structure of the hard template restricts the crystallization or aggregation of the precursors, and a mesoscopic phase having a structure opposite to that of the template can be obtained with the removal of template material by the appropriate method. Particles with different morphologies can be obtained by choosing hard templates with different structures.

\section{Soft Template}

The soft template does not have a fixed rigid structure. In the synthesis of nanoparticles, an aggregate with some certain structural features is formed by means of the intermolecular or intramolecular interaction force (hydrogen bonding, chemical bonding, and static electricity). Using these aggregates as a template, inorganic species are deposited on the surface or the interior of these templates by means of electrochemical method, precipitation, and other synthetic methods, forming particles with certain shape and size. Common soft template can be a surfactant, polymer and biopolymer, and so forth. The soft template has broad prospects for development in the synthesis of nanomaterials because of its advantages such as its good repeatability,

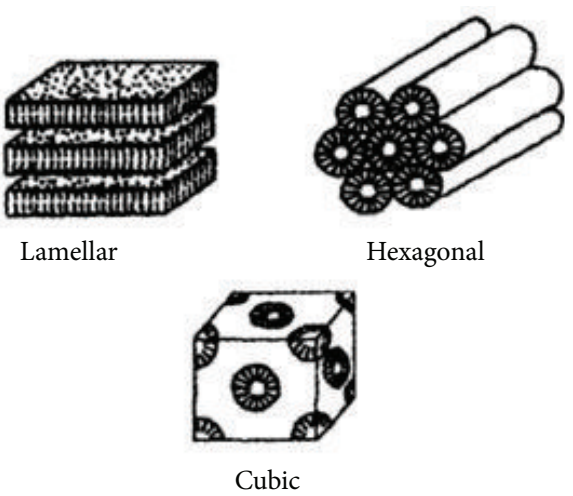

FIGURE 7: Structure of liquid crystals [49].

simplicity of the process, and no requirement for the removal of silicon [52, 54-57].

3.1. Surfactant. Surfactants are amphiphilic molecules, including ammonium salts, heterocyclic, carboxylic acid salts, sulfonate salts, and other ionic or nonionic surfactants. It is easy for the amphiphilic molecule groups to form a variety of ordered polymers in a solution, such as liquid crystals, vesicles, micelles, microemulsion, and self-assembled film $[58,59]$.

From the perspective of material chemistry, it is generally thought that the interaction between liquid crystal phase of surfactants and organic-inorganic interface plays a decisive role in the morphology of mesoporous materials $[60,61]$. In the strongly acidic environment, the interaction between the organic and inorganic interface is weak hydrogen bonds, while, in a strong alkaline environment, it is strong electrostatic attraction. Liquid crystalline phases formed by surfactants in the solution have various structures, such as lamellar phase, cubic phase, and hexagonal phase. It is also easy to construct and adjust [62]. Therefore, it is an ideal reactor for synthesizing mesoporous materials. The lamellar, cubic, and hexagonal structures are shown in Figure 7. 


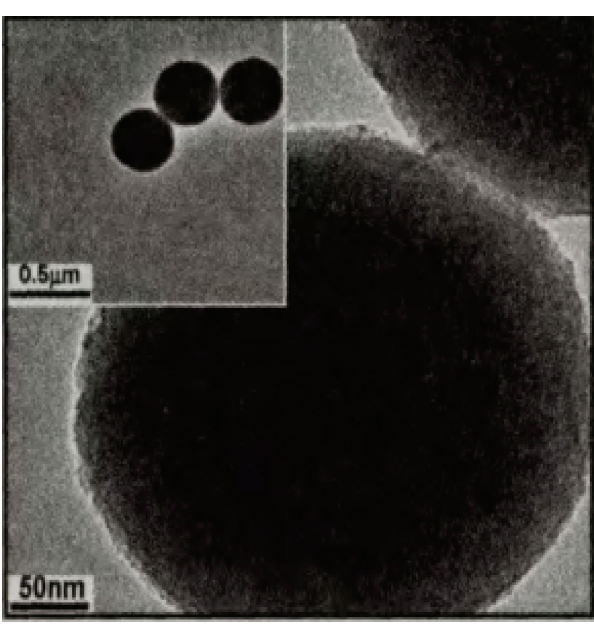

(a)

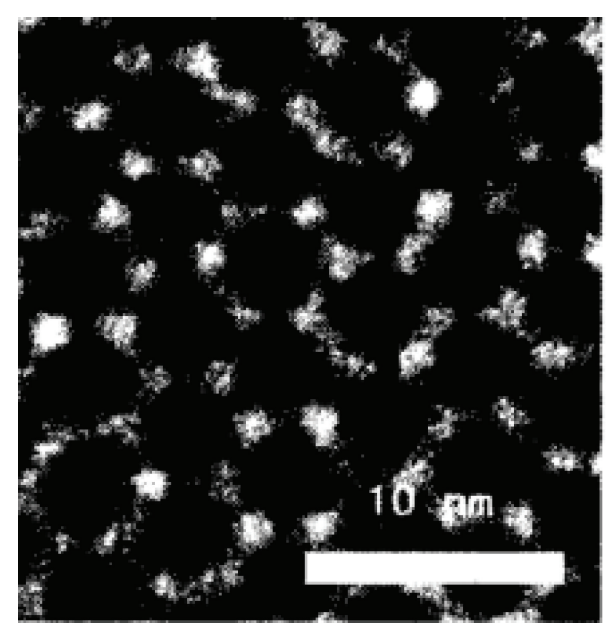

(b)

Figure 8: TEM image of mesoporous silica. (a) Silica microsphere. (b) Mesoporous silica thin film [50, 51].

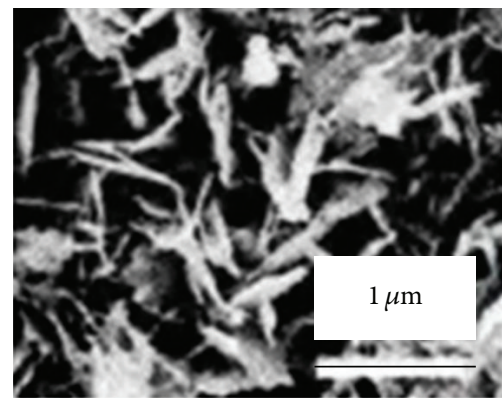

(a)

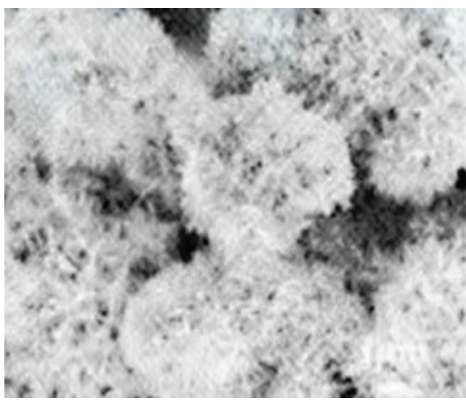

(b)

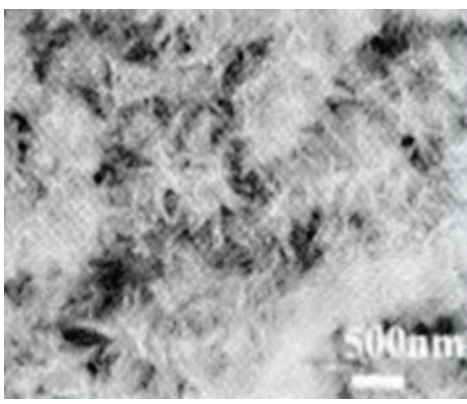

(c)

FIGURE 9: TEM image of cuprous oxide crystals. (a) Lamellar cuprous oxide crystal. (b) Urchin-like cuprous oxide crystal. (c) Acicular cuprous oxide crystal [52].

It is relatively easy to control the hydrolysis and polymerization of silicon species. Moreover, mesoporous silica with fibrous, thin film, tubular, spherical, and other different morphologies can be obtained by combining the collective effect of surfactants and the reaction environment. Zhang et al. [63] synthesized wormlike monodisperse mesoporous silica microspheres in neutral alcohol-water system by using laurylamine as surfactant and tetraethyl orthosilicate as silicon source. The silica microsphere is shown as Figure 8(a). The pore diameter can be regulated and controlled by adjusting the molar ratio and the concentration of the reactants and the ratio of alcohol/water. Wang et al. [53] synthesized mesoporous silica thin film in an acidic environment, which is shown in Figure 8(b), using tetraethyl orthosilicate as silicon source and cationic surfactant cetyltrimethylammonium bromide as template.

3.2. High Polymer. High polymer is often used as a template for the synthesis of mesoporous materials because of its large molecular weight, good stability, and diversity of molecular structures. The pore structure and the morphology play a decisive role in the application of mesoporous materials.
In recent years, researchers have emphasized the importance of controlling the morphology of mesoporous materials [64, 65], as different forms correspond to different application fields.

High polymer as a template and organic precursor form a certain liquid crystal structure by self-assembly method. In the fine crystal nucleation process, the selectivity of high polymers and certain crystal phase interact with each other, promoting or inhibiting the growth of crystal and thereby controlling and improving the morphology, size, and distribution of particles. Li et al. [66] successfully synthesized cuprous oxide crystals, which are lamellar, urchin-like, onedimensional line and acicular, in $\mathrm{CuSO}_{4}$ and $\mathrm{Na}_{2} \mathrm{SO}_{3}$ system by using polyacrylamide (PAM) as template and adjusting the concentration of $\mathrm{Cu}^{2+}$, PAM content, and temperature. The lamellar, urchin-like, and acicular cuprous oxides are shown as in Figure 9.

Block copolymer is one of the most important high polymers, which is connected by polymer chains with two or more different properties. During the process of synthesis of mesoporous materials, hydrogen bonding occurs as the hydrophilic segments and precursors interact with each other 
(a)

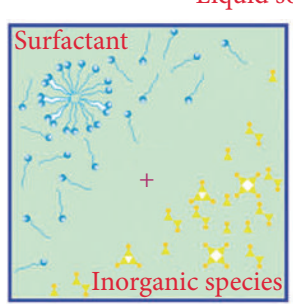

Cooperative nucleation

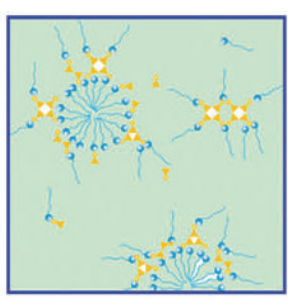

Cooperative aggregation and phase separation
Mixture of solution and precipitation
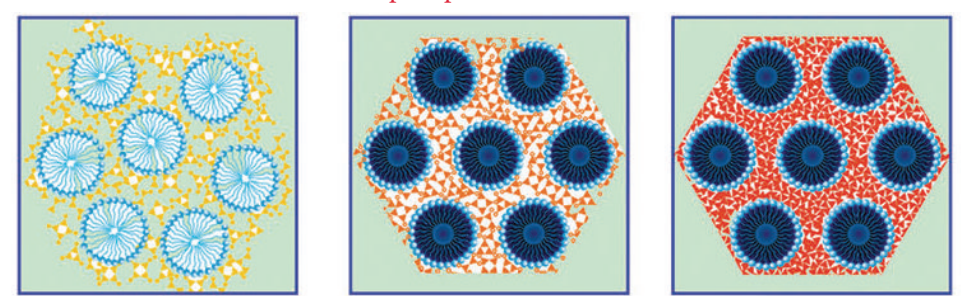

Liquid crystal formation with molecular inorganics
Further polymerization and condensation of inorganics

Template elimination

Mesoporous framework of final product

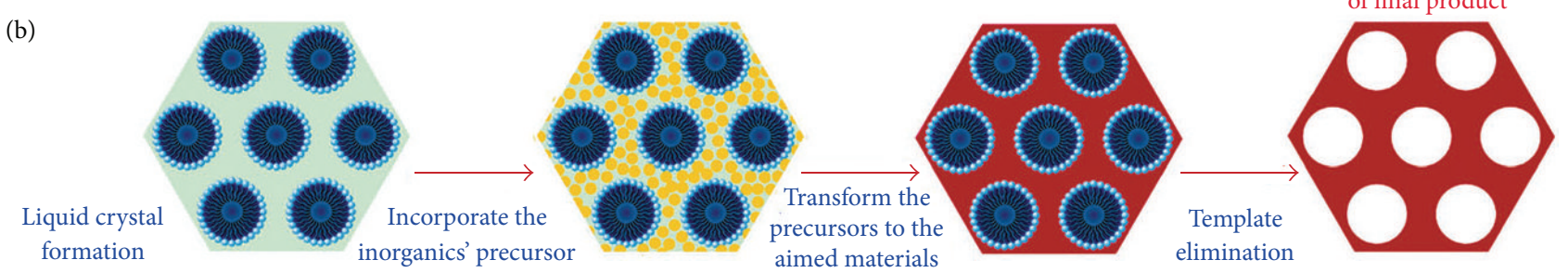

Figure 10: Mechanism of soft template method [53].

which results in the formation of the ordered mesoscopic phase. The pore diameter of the mesoscopic phase is determined by the hydrophobic chain of the block copolymer; therefore, the larger the molecular weight of hydrophobic segment is, the stronger the hydrophobic property is, and the larger the synthesized pore diameter is [67].

3.3. Biopolymer. Since biopolymer has the advantage of availability in wide variety of sources, its complex structure, nontoxicity and easy removal, and so forth, it has great potential to be used as a template in synthesis of other materials. By means of inducing the biomineralization of inorganic nanoparticles, biopolymer synthesizes nanomaterials that have certain structures.

Common biopolymers are DNA, proteins and polysaccharides, and so forth. Wang et al. [68], using linear DNA molecules of the same length as a template and controlling the synthesis of DNA sequences, synthesized petaloid and spherical gold nanoparticles. Zhang et al. [69] successfully synthesized hierarchical porous $\mathrm{SnO}_{2}$ nanomaterials that have a structure of cotton fibers by using cotton as a template and, respectively, adopting solvothermal method, ultrasonic method, and impregnation method.

3.4. Summary. Li and Zhao [70] schematically summarized two synthetic strategies of soft template method in Figure 10. There are two common mechanisms of the soft template: inorganic species and surfactants directly have supramolecular self-assembly, forming organic-inorganic phase. According to the combination of the two parts, it can be divided into electrostatic bonding, hydrogen bonding, and covalent bonding; since the interaction between inorganic species and surfactants is weak or they repel each other because they have identical electrical property, it is necessary to add the intermediate phase as a link to connect them, forming organic-inorganic phase.

In the self-assembly process, inorganic species interact with surfactants driven by Coulomb force, covalent bond, or hydrogen bonding. The interaction of the organic (surfactants)/inorganic (inorganic species) interface is a weak hydrogen bond force in the strong acid environment while it is a strong electrostatic attraction force in the strong alkaline environment. Thus inorganic species at the interface polymerize and cross-link and assemble with surfactants [71, 72].

\section{Comparison of Hard and Soft Template}

Hard template is mainly used some previously prepared template material, such as AAO template, mesoporous carbon, and polymer microsphere. It then applies for the physical or chemical growth or deposition of nanomaterials into nanopores of the template and finally removal the template; the dimensions and specifications of target production can be precisely controlled. Hence, hard template has high reproducibility and stability and is mostly used for synthesis of arrays of nanomaterial. However, the separation of template and production may cause damage to the structure of nanotubes, nanowire, or hollow balls.

On the other hand, soft template is mainly based on action of the micelle which forms organic-inorganic phase between surfactant, high polymer, and biopolymer and target production. The aggregation by weak intermolecular or intramolecular interaction creates a certain structure of space. Such aggregates have significant structural interface; it provides a unique interface to create a particular tendency 
of the distribution of inorganic spices and at last produce the nanomaterials with specific structures. Soft template is formed within the reaction, whereas hard template is prepared before the reaction. Hence, soft template is easier to build and remove than the hard template and it does not require complex equipment and strict production conditions, and the reaction has good controllability. Soft template is mostly used to produce various size, sharp structure of nanomaterials.

\section{Conclusions}

The rapid development of template method has promoted the innovation in nanotechnology by making the synthesis of nanomaterials with particular structure and morphology possible. Among these, hard template has a very vast advantage in the synthesis of particular nanomaterials since the hard template has adjustable pore structure and morphology. The sample obtained by this method has good dispersion, uniform pore size, and simple operation. Nevertheless, the hard template method also has disadvantages. The elimination of the template often leads to the collapse of part of the pore structure, affecting the performance of the product. Also, the obtained sample only shows order for smaller particle size. In addition, partially filled samples lead to discontinuous structure defects in the pore. The limited sources of template material restrict the widespread use of hard template method.

There are different types of soft templates. They only require simple equipment and have good reproducibility during the process of synthesis and the advantages of mass production. However, soft template method has also some disadvantages. The stability of soft template, which requires certain synthetic system, is not good. It can only form an ordered mesoscopic structure if the soft template has a strong interaction with the precursor. The precursor itself must be able to form a polymer structure with mechanical strength to certain degrees to guarantee that the structure will not collapse after the removal of the template. Furthermore, the mechanism of the soft template has not been unified for the nanomaterial synthesis.

Template method has a unique advantage in the synthesis of nanomaterials. To find an inexpensive, nontoxic template with good stability is a current hotspot in the research of template method. More studies are needed on the mechanism of the template by selecting the appropriate soft or hard template and changing the reaction conditions, which has a certain practical value.

\section{Competing Interests}

The authors declare that they have no competing interests.

\section{Acknowledgments}

This work was supported by the Natural Sciences and Engineering Research Council of Canada (NSERC) and the China Scholarship Council (Project no. 201307970002).

\section{References}

[1] H. Gleiter, "Nanostructured materials: basic concepts and microstructure," Acta Materialia, vol. 48, no. 1, pp. 1-29, 2000.

[2] Y. Huang and L. R. Zhang, "Research Status and Development Trend of nanomaterials preparation methods," Science and Technology Consulting Herald, vol. 10, p. 248, 2015.

[3] Y. Gao, S. Yao, J. Gong, and L. Qu, "Preparation of polyaniline nanotubes via 'thin glass tubes template' approach and its gas response," Macromolecular Rapid Communications, vol. 28, no. 3, pp. 286-291, 2007.

[4] Y. Xie, D. Kocaefe, Y. Kocaefe, J. Cheng, and W. Liu, “The effect of novel synthetic methods and parameters control on morphology of nano-alumina particles," Nanoscale Research Letters, vol. 11, article 259, 2016.

[5] T.-W. Kim, F. Kleitz, B. Paul, and R. Ryoo, "MCM-48-like large mesoporous silicas with tailored pore structure: facile synthesis domain in a ternary triblock copolymer-butanol-water system," Journal of the American Chemical Society, vol. 127, no. 20, pp. 7601-7610, 2005.

[6] I. Moriguchi, A. Ozono, K. Mikuriya, Y. Teraoka, S. Kagawa, and M. Kodama, "Micelle-templated mesophases of phenolformaldehyde polymer," Chemistry Letters, vol. 11, pp. 1171-1172, 1999.

[7] A. Dong, N. Ren, Y. Tang et al., "General synthesis of mesoporous spheres of metal oxides and phosphates," Journal of the American Chemical Society, vol. 125, no. 17, pp. 4976-4977, 2003.

[8] A. Firouzi, D. Kumar, L. M. Bull et al., "Cooperative organization of inorganic-surfactant and biomimetic assemblies," Science, vol. 267, no. 5201, pp. 1138-1143, 1995.

[9] Q. Huo, D. I. Margolese, U. Ciesla et al., "Generalized synthesis of periodic surfactant/inorganic composite materials," Nature, vol. 368, pp. 317-321, 1994.

[10] M. Zhou, Z. Wei, H. Qiao et al., "Particle size and pore structure characterization of silver nanoparticles prepared by confined arc plasma," Contributions to Atmospheric Physics, vol. 2009, no. 8, pp. 219-229, 2009.

[11] E. M. Johansson, J. M. Córdoba, and M. Odén, "Effect of heptane addition on pore size and particle morphology of mesoporous silica SBA-15," Microporous and Mesoporous Materials, vol. 133, no. 1-3, pp. 66-74, 2010.

[12] P. Innocenzi, S. Costacurta, T. Kidchob et al., "Mesoporous thin films: properties and applications," Chemical Society Reviews, no. 9, pp. 4198-4216, 2013.

[13] C. Boissiere, D. Grosso, S. Lepoutre, L. Nicole, A. B. Bruneau, and C. Sanchez, "Porosity and mechanical properties of mesoporous thin films assessed by environmental ellipsometric porosimetry," Langmuir, vol. 21, no. 26, pp. 12362-12371, 2005.

[14] K. Nielsch, J. Choi, K. Schwirn et al., "Self-ordering regimes of porous alumina: the 10 porosity rule," Nano Letters, vol. 2, no. 7, pp. 677-680, 2002.

[15] H. Tamon, H. Ishizaka, T. Yamamoto, and T. Suzuki, "Preparation of mesoporous carbon by freeze drying," Carbon, vol. 37, no. 12, pp. 2049-2055, 1999.

[16] N. Ren and Y. Tang, "Template-induced assembly of hierarchically ordered zeolite materials," Petrochemical Technology, vol. 34, no. 5, pp. 405-411, 2005.

[17] H. Masuda and K. Fukuda, "Ordered metal nanohole arrays made by a two-step replication of honeycomb structures of anodic alumina," Science, vol. 268, no. 5216, pp. 1466-1468, 1995. 
[18] J. Shang, L.-L. Liu, R.-M. Yang, and P.-X. Zhang, "Anodic aluminum oxide template with large area and small holes prepared by gradual voltage-rising method," Nanotechnology and Precision Engineering, vol. 6, no. 5, pp. 322-326, 2008.

[19] K. Liu, K. Du, J. Chen, L. Zhou, L. Zhang, and Y. Fang, "Fabrication of high quality ordered porous anodic aluminum oxide templates," High Power Laser and Particle Beams, vol. 22, no. 7, pp. 1531-1534, 2010.

[20] J. Fu, S. Cherevko, and C.-H. Chung, "Electroplating of metal nanotubes and nanowires in a high aspect-ratio nanotemplate," Electrochemistry Communications, vol. 10, no. 4, pp. 514-518, 2008.

[21] H. Zhuo, F. C. Peng, L. M. Lin, Y. Qu, and F. Lai, "Optical properties of porous anodic aluminum oxide thin films on quartz substrates," Thin Solid Films, vol. 519, no. 7, pp. 2308-2312, 2011.

[22] X. Ren, K. Senapati, W.-T. Jiang, and C.-H. Jiang, "Formation of ordered $\mathrm{TiO}_{2}$ nanostructural arrays with tunable shapes by magnetron sputtering method," Materials Chemistry \& Physics, vol. 126, no. 1-2, pp. 1-5, 2011.

[23] S. Öztürk, N. Klnç, N. Taşaltn, and Z. Z. Öztürk, "Fabrication of ZnO nanowires and nanorods," Physica E: Low-Dimensional Systems and Nanostructures, vol. 44, no. 6, pp. 1062-1065, 2012.

[24] W.-Y. Chang, J.-T. Wu, K.-H. Lin, S.-Y. Yang, K.-L. Lee, and P.K. Wei, "Fabrication of gold sub-wavelength pore array using gas-assisted hot embossing with anodic aluminum oxide (AAO) template," Microelectronic Engineering, vol. 88, no. 6, pp. 909913, 2011.

[25] J. W. Cui, Y. C. Wu, Y. Wang, H. Zheng, G. Xu, and X. Zhang, "A facile and efficient approach for pore-opening detection of anodic aluminum oxide membranes," Applied Surface Science, vol. 258, no. 14, pp. 5305-5311, 2012.

[26] P. Y. Loh, K. K. Lee, Y. Ng, C. H. Sow, and W. S. Chin, "Co/Al layered double hydroxides nanostructures: a binderless electrode for electrochemical capacitor," Electrochemistry Communications, vol. 43, pp. 9-12, 2014.

[27] N. Maleak, P. Potpattanapol, N. N. Bao et al., "Fabrication and magnetic properties of electrodeposited $\mathrm{Ni} / \mathrm{Cu}$ nanowires using the double bath method," Journal of Magnetism and Magnetic Materials, vol. 354, pp. 262-266, 2014.

[28] F. Li, L. Zhang, and R. M. Metzger, "On the growth of highly ordered pores in anodized aluminum oxide," Chemistry of Materials, vol. 10, no. 9, pp. 2470-2480, 1998.

[29] L. Sun, R. L. Zong, and J. Zhou, "Synthesis of ZnS nanowires by DC Electrodeposition in AAO template," Journal of Sichuan University (Natural Science Edition), vol. 2, no. 42, pp. 435-437, 2005.

[30] Q. Lu, F. Gao, S. Komarneni, and T. E. Mallouk, "Ordered SBA15 nanorod arrays inside a porous alumina membrane," Journal of the American Chemical Society, vol. 126, no. 28, pp. 8650-8651, 2004.

[31] P. Forrer, F. Schlottig, H. Siegenthaler, and M. Textor, "Electrochemical preparation and surface properties of gold nanowire arrays formed by the template technique," Journal of Applied Electrochemistry, vol. 30, no. 5, pp. 533-541, 2000.

[32] H.-Q. Li, J.-Y. Luo, X.-F. Zhou, C.-Z. Yu, and Y.-Y. Xia, "An ordered mesoporous carbon with short pore length and its electrochemical performances in supercapacitor applications," Journal of the Electrochemical Society, vol. 154, no. 8, pp. A731A736, 2007.
[33] X. Liu, L. Zhou, J. Li, Y. Sun, W. Su, and Y. Zhou, "Methane sorption on ordered mesoporous carbon in the presence of water," Carbon, vol. 44, no. 8, pp. 1386-1392, 2006.

[34] A. Vinu, K. Z. Hossain, G. S. Kumar, and K. Ariga, "Adsorption of L-histidine over mesoporous carbon molecular sieves," Carbon, vol. 44, no. 3, pp. 530-536, 2006.

[35] Z. Wen, J. Liu, and J. Li, "Core/shell Pt/C nanoparticles embedded in mesoporous carbon as a methanol-tolerant cathode catalyst in direct methanol fuel cells," Advanced Materials, vol. 20, no. 4, pp. 743-747, 2008.

[36] F. Su, J.Zeng, X. Bao, Y. Yu, J. Y. Lee, and X. S. Zhao, “Preparation and characterization of highly ordered graphitic mesoporous carbon as a Pt catalyst support for direct methanol fuel cells," Chemistry of Materials, vol. 17, no. 15, pp. 3960-3967, 2005.

[37] Y. Zhou, S. Ko, C. W. Lee, S. G. Pyo, S.-K. Kim, and S. Yoon, "Enhanced charge storage by optimization of pore structure in nanocomposite between ordered mesoporous carbon and nanosized $\mathrm{WO}_{3-\mathrm{x}}$," Journal of Power Sources, vol. 244, pp. 777782,2013

[38] L. Liu, Q.-F. Deng, B. Agula et al., "Synthesis of ordered mesoporous carbon materials and their catalytic performance in dehydrogenation of propane to propylene," Catalysis Today, vol. 186, no. 1, pp. 35-41, 2012.

[39] A. Vinu, K. Ariga, T. Mori et al., "Preparation and characterization of well-ordered hexagonal mesoporous carbon nitride," Advanced Materials, vol. 17, no. 13, pp. 1648-1652, 2005.

[40] C. Tang, L. Bombalski, M. Kruk, M. Jaroniec, K. Matyjaszewski, and T. Kowalewski, "Nanoporous carbon films from 'hairy' polyacrylonitrile-grafted colloidal silica nanoparticles," Advanced Materials, vol. 20, no. 8, pp. 1516-1522, 2008.

[41] H. Tamai, T. Kakii, Y. Hirota, T. Kumamoto, and H. Yasuda, "Synthesis of extremely large mesoporous activated carbon and its unique adsorption for giant molecules," Chemistry of Materials, vol. 8, no. 2, pp. 454-462, 1996.

[42] R. W. Pekala and D. W. Schaefer, "Structure of organic aerogels. 1. morphology and scaling," Macromolecules, vol. 26, no. 20, pp. 5487-5493, 1993.

[43] R. Zhang, Y. Lu, L. Zhan, X. Liang, G. Wu, and L. Ling, "Monolithic carbon aerogels from sol-gel polymerization of phenolic resoles and methylolated melamine," Carbon, vol. 41, no. 8, pp. 1660-1663, 2003.

[44] R. Zhang, W. Li, K. X. Li, C. Lu, L. Zhan, and L. Ling, "Effect of concentration of reactants on porosity of hydrogels, organic and carbon aerogels," Microporous and Mesoporous Materials, vol. 72, no. 1-3, pp. 167-173, 2004.

[45] H. W. Wang, X. Yin, Y. Z. Long et al., "Discussion on one step synthesis of mesoporous carbon materials," Experimental Technology and Management, vol. 6, no. 31, pp. 48-53, 2014.

[46] J. Roggenbuck and M. Tiemann, "Ordered mesoporous magnesium oxide with high thermal stability synthesized by exotemplating using CMK-3 carbon," Journal of the American Chemical Society, vol. 127, no. 4, pp. 1096-1097, 2005.

[47] J. Roggenbuck, G. Koch, and M. Tiemann, "Synthesis of mesoporous magnesium oxide by CMK-3 carbon structure replication," Chemistry of Materials, vol. 18, no. 17, pp. 4151-4156, 2006.

[48] A. Sakthivel, S.-J. Huang, W.-H. Chen et al., "Replication of mesoporous aluminosilicate molecular sieves (RMMs) with zeolite framework from mesoporous carbons (CMKs)," Chemistry of Materials, vol. 16, no. 16, pp. 3168-3175, 2004. 
[49] Z. X. Yang, Y. D. Xia, and R. Mokaya, "Zeolite ZSM-5 with unique supermicropores synthesized using mesoporous carbon as a template," Advanced Materials, vol. 16, no. 8, pp. 727-732, 2004.

[50] K. Zhang, Q. Fu, Y. H. Huang et al., "Study on preparation of monodisperse polystyrene microshperes with designable size," Ion Exchange and Adsorption, vol. 22, no. 2, pp. 140-145, 2006.

[51] L. Lin, "Preparation of $\mathrm{ZnS} / \mathrm{CdS}$ hollow spheres using poly(styrene-acrylic acid) latex particles as template," Liaoning Chemical Industry, vol. 37, no. 11, pp. 739-741, 2008.

[52] J. Lee, J. Kim, and T. Hyeon, "Recent progress in the synthesis of porous carbon materials," Advanced Materials, vol. 18, no. 16, pp. 2073-2094, 2006.

[53] X. L. Wang, B. Zhou, Z. Xu et al., "Preparation and $\mathrm{TiO}_{2}$ assembly of high ordered mesoporous carbon films," Atomic Energy Science and Technology, vol. 40, pp. 55-60, 2006.

[54] Y. Wan, Y. Shi, and D. Zhao, "Designed synthesis of mesoporous solids via nonionic-surfactant-templating approach," Chemical Communications, vol. 38, no. 28, pp. 897-926, 2007.

[55] A.-H. Lu and F. Schüth, "Nanocasting: a versatile strategy for creating nanostructured porous materials," Advanced Materials, vol. 18, no. 14, pp. 1793-1805, 2006.

[56] Y. Lu, "Surfactant-templated mesoporous materials: from inorganic to hybrid to organic," Angewandte Chemie-International Edition, vol. 45, no. 46, pp. 7664-7667, 2006.

[57] Y. Wan, H. Yang, and D. Zhao, "'Host-guest' chemistry in the synthesis of ordered nonsiliceous mesoporous materials," Accounts of Chemical Research, vol. 39, no. 7, pp. 423-432, 2006.

[58] Q. Huo, D. I. Margolese, U. Ciesla et al., "Generalized synthesis of periodic surfactant/inorganic composite materials," Nature, vol. 368, no. 6469, pp. 317-321, 1994.

[59] M. F. Islam, E. Rojas, D. M. Bergey, A. T. Johnson, and A. G. Yodh, "High weight fraction surfactant solubilization of singlewall carbon nanotubes in water," Nano Letters, vol. 3, no. 2, pp. 269-273, 2003.

[60] D. Zhao, Y. Peidong, H. Qisheng, C. Bradley F, and S. Galen D, "Topological construction of mesoporous materials," Current Opinion in Solid State and Materials Science, vol. 3, no. 1, pp. 111$121,1998$.

[61] H.-P. Lin and C.-Y. Mou, "Structural and morphological control of cationic surfactant-templated mesoporous silica," Accounts of Chemical Research, vol. 35, no. 11, pp. 927-935, 2002.

[62] L. Y. Wang and S. S. Liao, Liquid Crystal Chemistry, Science Press, 1998.

[63] X. L. Zhang, W. Y. Zhu, Q. Cai et al., "A size-controllable preparation of monodispers emesoporous $\mathrm{SiO}_{2}$ microspheres," Journal of Functional Materials, vol. 42, pp. 803-808, 2011.

[64] M.-C. Chao, D.-S. Wang, H.-P. Lin, and C.-Y. Mou, "Control of single crystal morphology of SBA-1 mesoporous silica," Journal of Materials Chemistry, vol. 13, no. 12, pp. 2853-2854, 2003.

[65] Y. K. Hwang, J.-S. Chang, Y.-U. Kwon, and S.-E. Park, "Microwave synthesis of cubic mesoporous silica SBA-16," Microporous \& Mesoporous Materials, vol. 68, no. 1-3, pp. 2127, 2004.

[66] R. Li, L.-M. Yu, L.-N. Jia, X.-F. Yan, and L. Dong, "Biomimetic synthesis of different morphology $\mathrm{Cu}_{2} \mathrm{O}$ induced by using polymer as template," Chinese Journal of Inorganic Chemistry, vol. 29, no. 2, pp. 265-270, 2013.

[67] C.-Z. Yu, J. Fan, and D.-Y. Zhao, "Block copolymer synthesis of high-quality cubic, large pore mesoporous millimeter spheres in the presence of inorganic salts," Acta Chimica Sinica, vol. 60, no. 8, pp. 1357-1360, 2002.
[68] Z. Wang, J. Zhang, J. M. Ekman, P. J. A. Kenis, and Y. Lu, "DNA-mediated control of metal nanoparticle shape: one-pot synthesis and cellular uptake of highly stable and functional gold nanoflowers," Nano Letters, vol. 10, no. 5, pp. 1886-1891, 2010.

[69] C. L. Zhang, J. Wang, and R. J. Hu, "Biotemplated systhesis of porous and hierarchy $\mathrm{SnO}_{2}$ and sensitivity property for ethanol," New Chemical Materials, vol. 43, no. 1, pp. 99-101, 2015.

[70] W. Li and D. Zhao, "An overview of the synthesis of ordered mesoporous materials," Chemical Communications, vol. 49, no. 10, pp. 943-946, 2013.

[71] N. R. Jana, L. Gearheart, and C. J. Murphy, "Seed-mediated growth approach for shape-controlled synthesis of spheroidal and rod-like gold nanoparticles using a surfactant template," Advanced Materials, vol. 13, no. 18, pp. 1389-1393, 2001.

[72] D. Zhao, Q. Huo, J. Feng, B. F. Chmelka, and G. D. Stucky, "Nonionic triblock and star diblock copolymer and oligomeric sufactant syntheses of highly ordered, hydrothermally stable, mesoporous silica structures," Journal of the American Chemical Society, vol. 120, no. 24, pp. 6024-6036, 1998. 

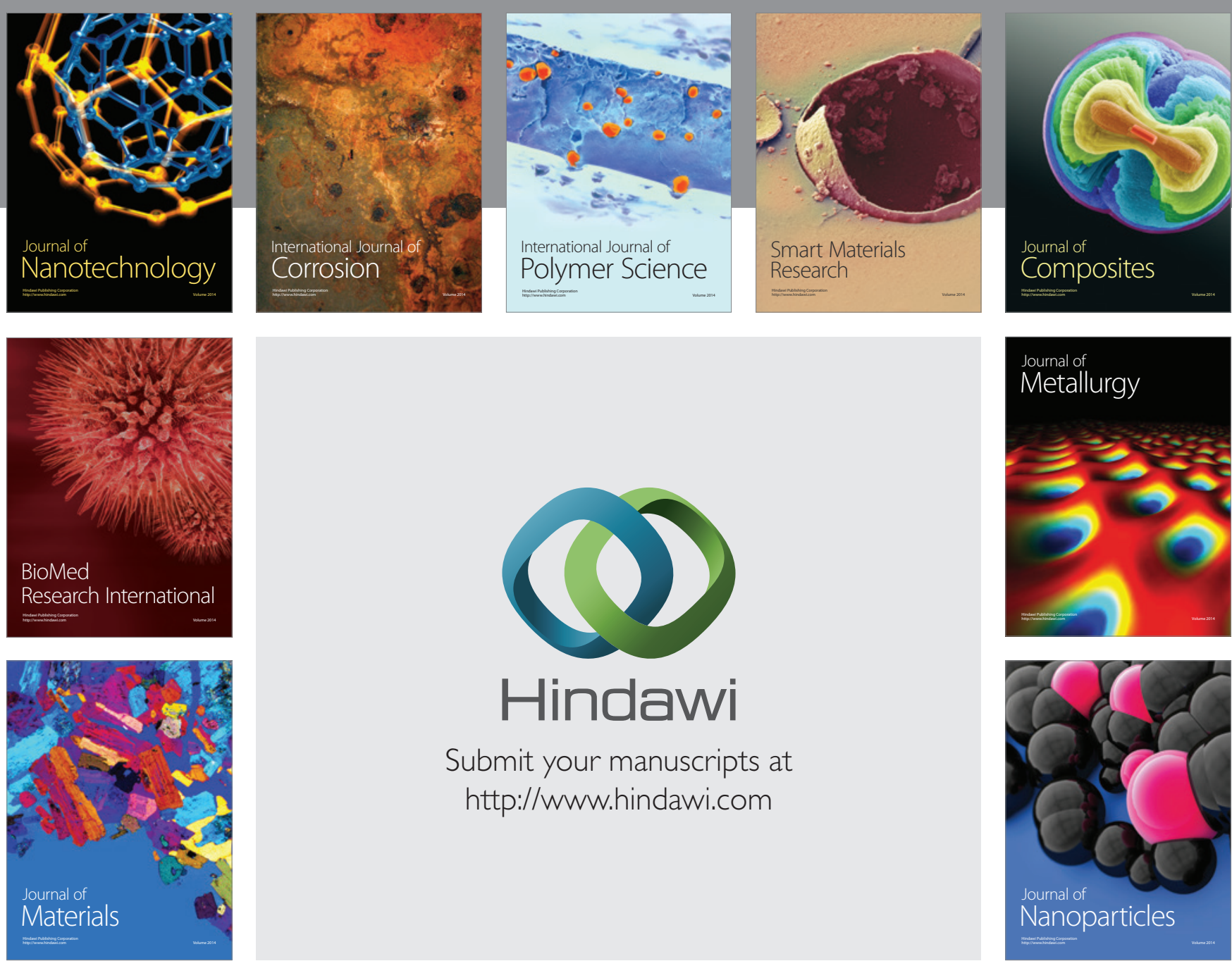

\section{Hindawi}

Submit your manuscripts at

http://www.hindawi.com

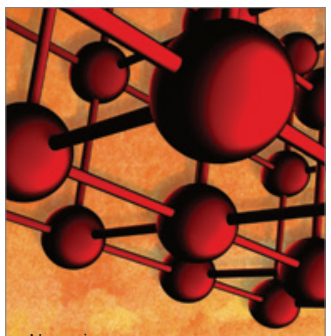

Materials Science and Engineering
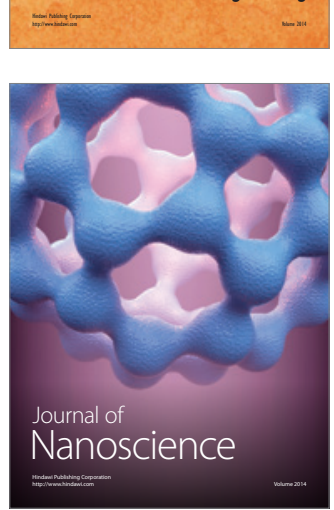
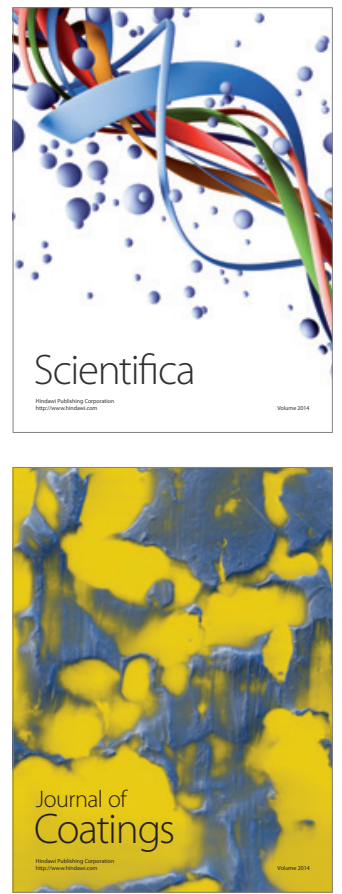
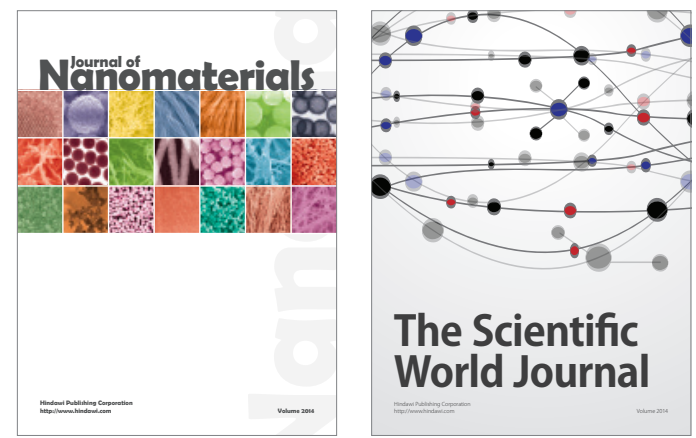

The Scientific World Journal
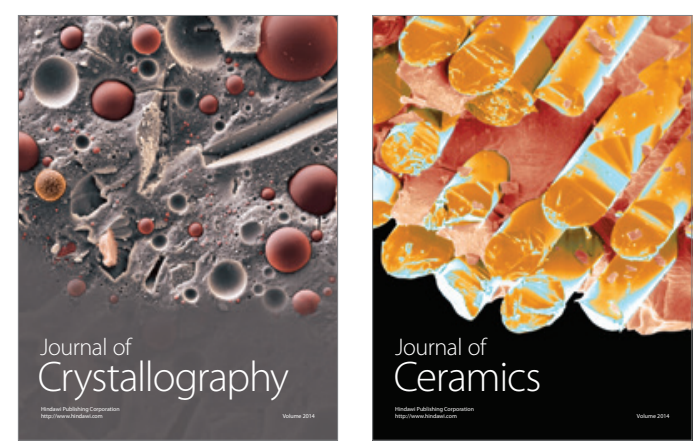
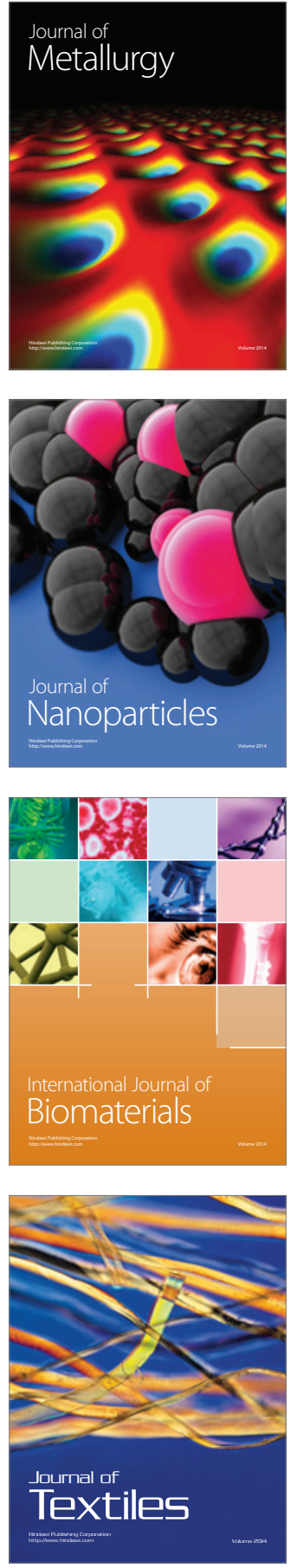